\title{
Localization of ultrasound in a three-dimensional elastic network
}

\author{
HEFEl HU ${ }^{1 *}$, A. STRYBULEVYCH ${ }^{1}$, J. H. PAGE ${ }^{1 \dagger}$, S. E. SKIPETROV² AND B. A. VAN TIGGELEN² \\ ${ }^{1}$ Department of Physics and Astronomy, University of Manitoba, Winnipeg, Manitoba, R3T 2N2, Canada \\ ${ }^{2}$ Université Joseph Fourier, Laboratoire de Physique et Modélisation des Milieux Condensés, CNRS, 25 Rue des Martyrs, BP 166, 38042 Grenoble, France \\ *Present address: Department of Physics, University of Illinois at Urbana-Champaign, Urbana, Illinois 61801-3080, USA \\ †e-mail: jhpage@cc.umanitoba.ca
}

After exactly half a century of Anderson localization ${ }^{1}$, the subject is more alive than ever. Direct observation of Anderson localization of electrons was always hampered by interactions and finite temperatures. Yet, many theoretical breakthroughs were made, highlighted by finite-size scaling ${ }^{2}$, the self-consistent theory ${ }^{3}$ and the numerical solution of the Anderson tight-binding model ${ }^{4,5}$. Theoretical understanding is based on simplified models or approximations and comparison with experiment is crucial. Despite a wealth of new experimental data, with microwaves and light $^{6-12}$, ultrasound ${ }^{13}$ and cold atoms $^{14-16}$, many questions remain, especially for three dimensions. Here, we report the first observation of sound localization in a random three-dimensional elastic network. We study the time-dependent transmission below the mobility edge, and report 'transverse localization' in three dimensions, which has never been observed previously with any wave. The data are well described by the self-consistent theory of localization. The transmission reveals non-Gaussian statistics, consistent with theoretical predictions.

Most text books on condensed matter explain that the electronic states in disordered conductors are extended plane or Bloch waves with finite lifetimes. This gives rise to 'ohmic resistance', proportional to the length of the sample. In the picture presented by Anderson ${ }^{1}$, 'large' disorder makes electronic states localized in space. This offers a mechanism to explain the widely observed metal-insulator transitions ${ }^{17}$. Scaling theory proposes a single parameter, the Thouless conductance $g$, to describe the anomalous length dependence of the resistance of a sample ${ }^{2}$. The localized regime is characterized by the so-called Thouless criterion $g<1$. Although these ideas were first proposed for electron localization, in the early 1980s interest in classical-wave localization was raised ${ }^{18,19}$, with the promise of avoiding the difficulties caused by interactions in electronic systems. At the same time, the absence of bound states for classical waves makes localization more challenging to achieve in practice, with absorption as an extra concern.

Here, we demonstrate Anderson localization of ultrasound in a three-dimensional (3D) medium. Our samples are single-component random networks made by brazing aluminium beads together, see Fig. la. With ultrasound, we probe the vibrational excitations of the network in the intermediate frequency regime $(0.2-3 \mathrm{MHz})$, where the wavelength is comparable to the bead and pore sizes. We use pulsed techniques to measure the amplitude transmission coefficient, shown in Fig. 1b. The transmission spectrum exhibits a succession of bandgaps and a
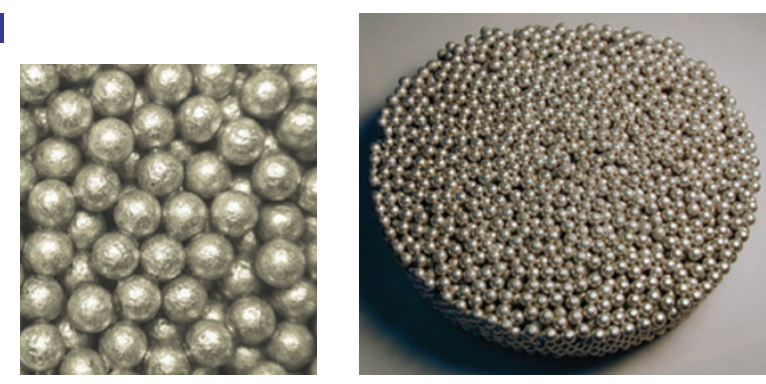

b

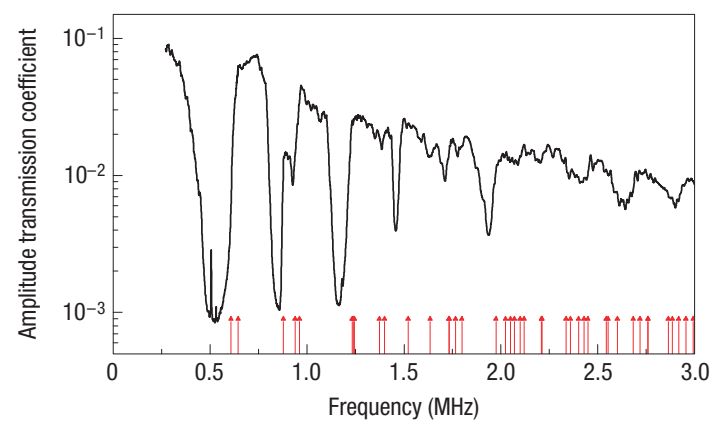

Figure 1 The random elastic network and corresponding transmission coefficient. a, Top view of one of the samples (right) and a magnified image showing the mesoscale structure of the random elastic network (left). The samples were made from $4.11 \pm 0.03 \mathrm{~mm}$ diameter aluminium beads brazed together at a volume fraction of approximately $55 \%$. The diameter $(120 \mathrm{~mm})$ of the slab-shaped samples was much larger than the thickness $L$, which ranged from 8.3 to $23.5 \mathrm{~mm}$. b. Frequency dependence of the amplitude transmission coefficient for $L=14.5 \mathrm{~mm}$. The arrows indicate the resonance frequencies of isolated aluminium beads.

pass bands, due to the overlapping resonances of the aluminium beads ${ }^{20}$. Our study focuses on frequencies just below the first bandgap at $0.5 \mathrm{MHz}$, and at higher frequencies $(1.6-3 \mathrm{MHz})$, where it was possible to extract the phase and amplitude of the coherent pulse for longitudinal waves crossing the sample. Hence, we were able to measure the longitudinal phase $v_{\mathrm{p}}$ and group $v_{\mathrm{g}}$ velocities, as well as the scattering mean free path $\ell$ (refs 21,22). Note that because absorption is negligible (see below), the attenuation of the coherent pulse gives $\ell$ directly. Around 
a

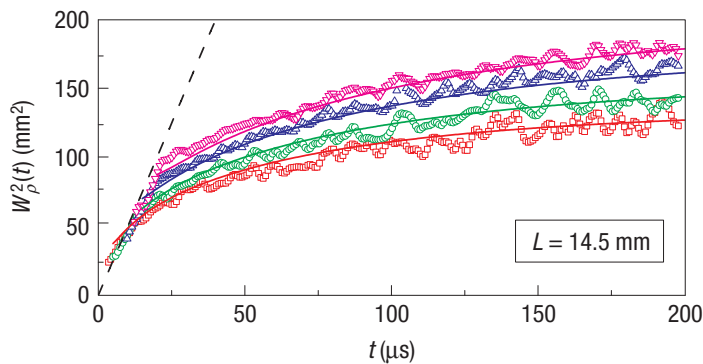

b

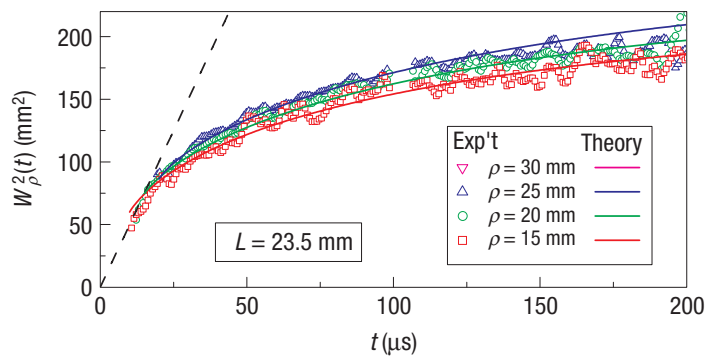

$\mathbf{G}$

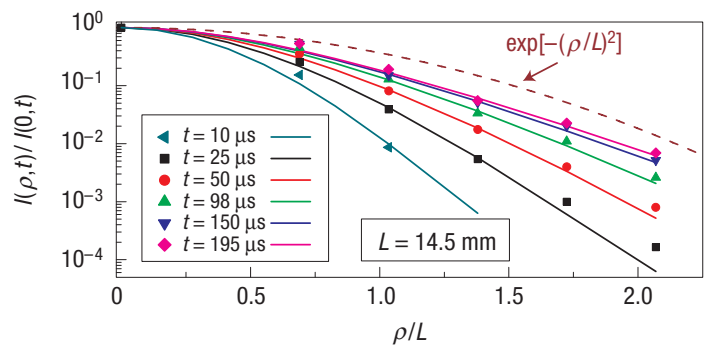

Figure 2 Transverse localization in three dimensions. a,b, Temporal evolution of the effective width squared, $w_{\rho}^{2}(t)$, of the transmitted intensity emanating from a point source for several transverse displacements $\rho$ of the detector and for two sample thicknesses. The frequency is $2.4 \mathrm{MHz}$. The solid curves are the best fits of our self-consistent theory to the experimental data (symbols), from which we determine $\ell_{\mathrm{B}}^{*}=2 \mathrm{~mm}, D_{\mathrm{B}}=11 \mathrm{~m}^{2} \mathrm{~s}^{-1}, \xi \approx 15 \mathrm{~mm}$ for the $L=14.5 \mathrm{~mm}$ sample and $\xi \approx 7 \mathrm{~mm}$ for the $L=23.5 \mathrm{~mm}$ sample. Other input parameters- $k \ell=1.8$ and the internal reflection coefficient $R=0.82$-were obtained from independent measurements. The dashed line shows the linear time dependence of $w_{\rho}^{2}$ that would be seen for diffuse waves, using $D=1.25 \mathrm{~m}^{2} \mathrm{~s}^{-1}$. c, Dependence of the intensity ratio on distance $\rho$ at six different times, showing the non-Gaussian profile that is found both experimentally (symbols) and theoretically (solid curves).

$0.20 \mathrm{MHz}, v_{\mathrm{p}}=1.75 \mathrm{~km} \mathrm{~s}^{-1}, v_{\mathrm{g}}=2.1 \mathrm{~km} \mathrm{~s}^{-1}$ and $\ell=2.2 \mathrm{~mm}$, whereas around $2.4 \mathrm{MHz}, v_{\mathrm{p}}=5.0 \mathrm{~km} \mathrm{~s}^{-1}, v_{\mathrm{g}}=5.2 \mathrm{~km} \mathrm{~s}^{-1}$ and $\ell=0.6 \mathrm{~mm}$. This leads to a product of wave vector $k$ and scattering mean free path $k \ell=1.6$ at $0.20 \mathrm{MHz}$ and $k \ell=1.8$ at $2.4 \mathrm{MHz}$. The values of $k \ell$ for shear waves, likely to dominate, are not known but are probably roughly equal ${ }^{23}$. The small values of $k \ell$ indicate that our samples are strongly scattering. For 3D disorder, localization is expected when $k \ell \lesssim 1$ (ref. 24). Because the exact critical value is not known, the ultrasonic waves at both frequencies are candidates to be Anderson localized.

In previous reports on Anderson localization with classical waves, absorption has been a major obstacle to reaching unambiguous conclusions $s^{7,8,10,11,25}$. The following experiment is capable of probing Anderson localization without being blurred by absorption. We measure the spatially and time-resolved transmitted intensity through our sample. Using a quasi-point source that is about a wavelength wide and a subwavelength-diameter detector that scans the sample at various transverse positions $\rho$ in the a
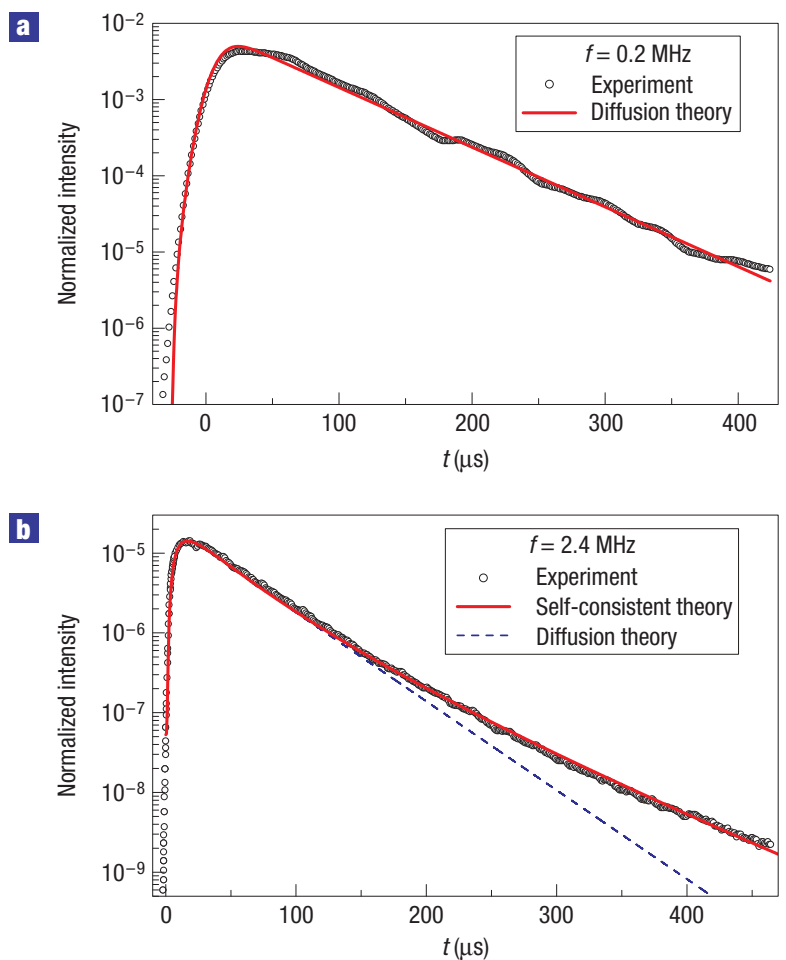

Figure 3 Averaged time-dependent transmitted intensity. Transmitted intensity, $I(t)$, normalized so that the peak of the input pulse is unity and centred on $t=0$, at representative frequencies in the diffuse (a) and localized (b) regimes for the $L=14.5 \mathrm{~mm}$ sample investigated in Fig. 2a,c. In a, the fit to the diffusion theory with $R=0.85$ gives $D=3.0 \mathrm{~m}^{2} \mathrm{~s}^{-1}, \ell^{*} \simeq 2.5 \mathrm{~mm}$ and $\tau_{\mathrm{a}}$ too large to be measurable. In $\mathbf{b}$, the data are fitted by the self-consistent theory (red curve), with $\xi=15 \mathrm{~mm}, \ell_{\mathrm{B}}^{*}=2 \mathrm{~mm}, D_{\mathrm{B}}=16 \mathrm{~m}^{2} \mathrm{~s}^{-1}$ and $\tau_{\mathrm{a}}=160 \mu \mathrm{s}$. For comparison, the dashed blue line shows the long-time behaviour predicted by diffusion theory.

near field ( $\rho=0$ opposite to the source), the average transmitted intensity $I(\rho, t)$ was determined ${ }^{26}$. From these measurements, we determine the ratio $I(\rho, t) / I(0, t)$, probing the dynamic spreading of the intensity in the transverse direction. Any possible absorption factor $\exp \left(-t / \tau_{a}\right)$ cancels in this ratio. For each $\rho$, a transverse width $w_{\rho}(t)$ of $I(\rho, t)$ can be defined by setting $I(\rho, t) / I(0, t) \equiv \exp \left[-\rho^{2} / w_{\rho}^{2}(t)\right]$. If the wave propagation is diffuse, the spatial intensity profile is Gaussian and $w_{\rho}^{2}(t)=4 D t$ is independent of $\rho$. We have observed this normal diffuse behaviour in less strongly scattering samples, and hence have been able to demonstrate a way of measuring the diffusion coefficient without the usual complications due to boundary conditions and absorption ${ }^{26}$.

For frequencies between 1.6 and $3 \mathrm{MHz}$, we observe markedly different behaviour, shown in Fig. 2 for two representative samples at $2.4 \mathrm{MHz}$. Instead of increasing linearly with propagation time, $w_{\rho}^{2}(t)$ is seen to saturate. This saturation is reminiscent of transverse localization, previously predicted in 3D systems with $2 \mathrm{D}$ disorder ${ }^{27}$ and observed in 2D disordered photonic crystals ${ }^{12}$. In our samples, the disorder is clearly $3 \mathrm{D}$ because the scattering mean free path is much smaller than the sample thicknesses $(10<L / \ell<40)$. It is not at all clear a priori that transverse localization can occur with $3 \mathrm{D}$ disorder. Scaling theory ${ }^{2}$ predicts a diffusivity $D(L)$ dependent on the sample size $L$, so that $w_{\rho}^{2}(t)=4 D(L) t$ still rises linearly with time. The saturation of $w_{\rho}(t)$ could possibly be explained by a diffusivity $D(t)$ decaying 
a
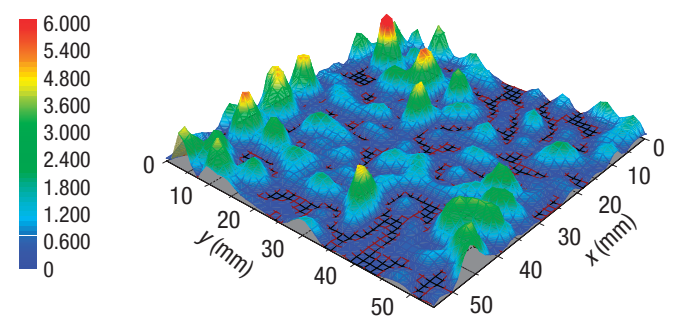

G

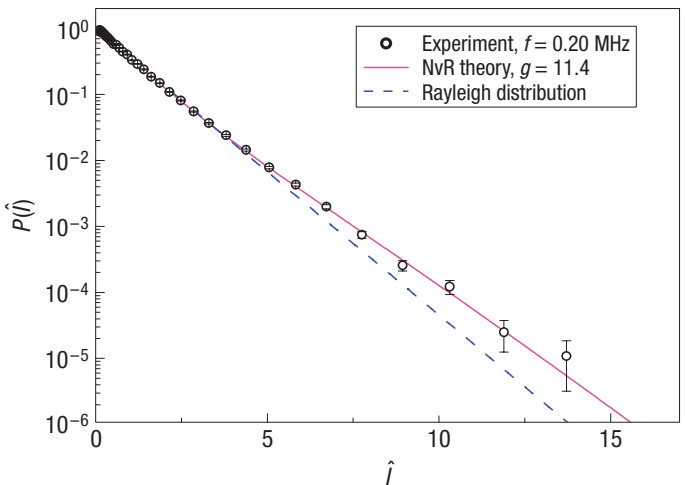

b

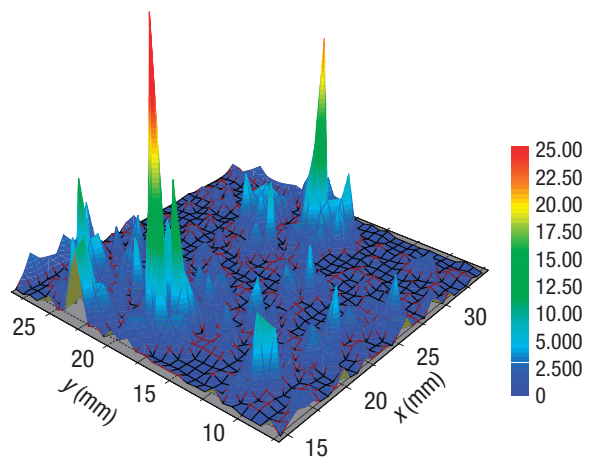

d

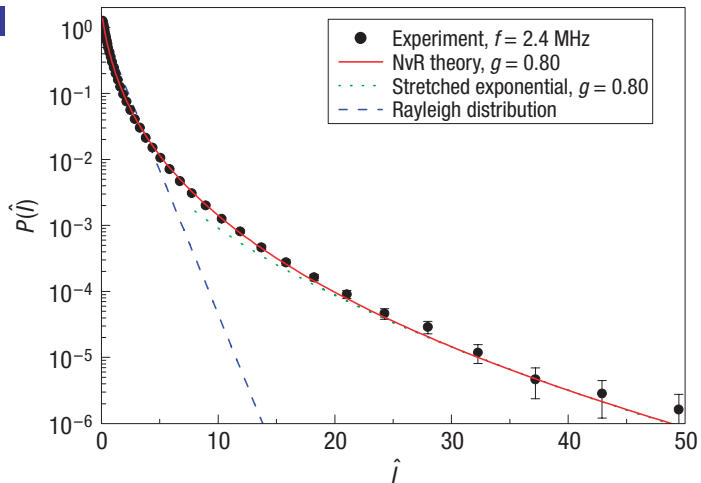

Figure 4 Statistical approach to localization. Comparison of the near-field speckle patterns $I(x, y) /\langle I\rangle$ for diffuse and localized waves observed at frequencies of 0.20 (a) and $2.4 \mathrm{MHz}$ (b). In a, the speckle pattern is typical for the diffuse regime, whereas $\mathbf{b}$ reveals the narrow intense spikes that we explain in terms of Anderson localization. Note the different colour scales in the two figures. c, The measured probability distribution $P(\hat{I})$ of normalized intensity $\hat{I}=I /\langle I\rangle$ at $0.2 \mathrm{MHz}$ (open circles) is close to the Rayleigh distribution (dashed blue line). The experimental error bars are proportional to the square root of the number of counts in each bin. The solid magenta curve is the best fit to the theory of ref. 30 with $g=11.4$. d, At $2.4 \mathrm{MHz}, P(\hat{I})$ (filled symbols) shows very large departures from the Rayleigh distribution. The error bars represent the error in the mean of experimental data for several samples. The solid curve shows the theory ${ }^{30}$ with $g=0.80$. At large $\hat{l} \gtrsim 25$, the data can also be described by a stretched exponential $P(\hat{I}) \propto \exp (-2 \sqrt{g \hat{l}})$ with the same value of $g$ (dotted curve). The large fluctuations $\left\langle\hat{l}^{2}\right\rangle=2.74$ and the large deviation from Rayleigh statistics with $g<1$ support our conclusion that Anderson localization of sound has set in at frequencies near $2.4 \mathrm{MHz}$.

with time $e^{9-11}$. This would still lead to a Gaussian transverse intensity profile and hence to $w_{\rho}^{2}$ constant with $\rho$. However, this is not what we observe.

To describe the dynamics of the anomalous transverse confinement of the multiply scattered waves, we apply a novel version of the self-consistent theory of localization. The new element consists of incorporating boundary conditions self-consistently ${ }^{28,29}$. This theory provides a position-dependent, dynamic diffusivity kernel $D\left(\mathbf{r}, t-t^{\prime}\right)$. Near the mobility edge, the position dependence of $D$ affects all aspects of wave transport considerably. The self-consistent theory requires as input the value for $k \ell$, the localization length $\xi$, the diffusion constant $D_{\mathrm{B}}$ free from macroscopic interferences and the internal reflection coefficient $R$. In the model, we replace the incident focused beam by a point source at depth $\ell_{\mathrm{B}}^{*}$. This is the transport mean free path associated with diffusion in the absence of macroscopic interferences, which ought to be negligible just after the beam comes in. Figure 2 compares the observed dynamics of transverse width to this theory. Excellent agreement with the data is seen for all $\rho$ with a single set of parameters for each sample (solid curves), yielding, in particular, $\xi \approx 15 \mathrm{~mm}$ and $\xi \approx 7 \mathrm{~mm}$ for the thinner and thicker samples respectively. We attribute the smaller value of $\xi$ for the thicker sample to stronger scattering due to small differences in the microstructure. This is consistent with the strong sensitivity of $\xi$ to small changes in disorder that is expected near the localization transition. As $\rho$ increases, the curves $w_{\rho}^{2}(t)$ move upwards, meaning that the observed transverse profile $I(\rho, t)$ is not Gaussian. Figure 2 shows that this behaviour is well captured by the theory, in which the position dependence of $D$ is a crucial element. Any homogeneous absorption would not affect the results in Fig. 2. We believe that this combination of theory and experiment provides strong evidence for Anderson localization of ultrasound near $2.4 \mathrm{MHz}$ in our samples and enables us to estimate the mobility edge $(k \ell)_{c}$, which we find to be approximately $1 \%$ above the measured $k \ell=1.8$.

To find extra support for our conclusions, we have measured the time-dependent transmission using an extended quasi-plane-wave source. Near $0.2 \mathrm{MHz}$, the average transmitted intensity $I(t)$ was found to decay exponentially at long times (Fig. 3a), with the entire time dependence of $I(t)$ being well described by diffusion theor ${ }^{26}$. We conclude that multiply scattered ultrasound at $0.2 \mathrm{MHz}$ propagates in a normal, diffuse way. In contrast, for ultrasound propagating near $2.4 \mathrm{MHz}$, the time dependence of $I(t)$ shows a quite different behaviour (Fig. 3b), with a non-exponential tail at long times. Similar behaviour has been reported before and is often explained by a time-dependent reduction of the effective diffusion coefficient $D(t)$ (refs 9-11). The solid curve in Fig. $3 \mathrm{~b}$ is a fit to the self-consistent theory, and gives an excellent description of the experiment at all propagation times. The good agreement between theory and experiment supports our previous conclusion that ultrasound at $2.4 \mathrm{MHz}$ is localized. From the fits in Figs 2 and $3 \mathrm{~b}$ and the relation $D_{\mathrm{B}}=v_{E} \ell_{\mathrm{B}}^{*} / 3$, 
we find transport velocities 3-5 times larger than the phase velocity of longitudinal waves. Further theoretical work is needed to understand these-apparently large-values for $v_{E}$ in the localized regime.

We also address the statistical approach to Anderson localization $^{7}$. The normalized transmitted intensity $\hat{I} \equiv I /\langle I\rangle$ was measured for a large number of individual speckle spots in the near field, and for a broad incident beam. The intensity distributions $P(\hat{I})$ for both frequencies are shown in Fig. 4 . We have compared our data to the theory of ref. 30, with the Thouless conductance $g$ as the only free parameter in the fit. The agreement is excellent for $g=0.80 \pm 0.08$ at $2.4 \mathrm{MHz}$ and $g=11.4 \pm 0.8$ at $0.2 \mathrm{MHz}$. The strong deviation from Rayleigh statistics with $g<1$ observed at $2.4 \mathrm{MHz}$ is interpreted as a signature of localization ${ }^{7}$. The remarkable agreement with theory, derived for the intensity in the far field, and for $g \gg 1$, reveals a universality of the statistics of localized waves.

Our discovery of 3D transverse localization provides a powerful new approach for guiding future investigations of localization for any type of wave-not only for assessing whether or not the waves are localized but also for measuring the localization length. By studying three different fundamental aspects of Anderson localization simultaneously, we have also shown the versatility of ultrasonic experiments, which are very well suited for undertaking a complete study of this phenomenon-one of the most fascinating in all condensed matter. Our results suggest that it should now be feasible to determine critical exponents, by measuring the variation of the localization length with frequency near the mobility edge, as well as to investigate the sensitivity of the localization length to sample microstructure, the spatial correlations of localized waves in $3 \mathrm{D}$ and the microscopic nature of localized states. All of these experiments, either with sound or light, would make unique contributions to advancing our understanding of Anderson localization.

\section{METHODS}

The elastic network of aluminium beads was created by precisely controlling the flux, alloy concentration and temperature during brazing to form elastic bonds between the beads while preserving their spherical shape. The samples were made in the form of disc-shaped slabs, to minimize edge effects. After brazing, the samples were lightly polished to ensure that opposite faces of the slabs were flat and parallel, and carefully cleaned to remove any residue of the brazing process. The samples were waterproofed with very thin plastic walls to enable pulsed ultrasonic immersion transducer techniques to be used ${ }^{26}$, thereby ensuring that the samples remained dry when immersed in a water tank between the generating and detecting transducers. The coherent pulse, from which $v_{\mathrm{p}}, v_{\mathrm{g}}$ and $\ell$ were determined, was then measured by scanning the position of the sample in a plane parallel to the sample surface and averaging the transmitted field over all positions ${ }^{21,22}$. These measurements were made using large-diameter immersion transducers to aid in the spatial averaging of the transmitted field and hence in the extraction of the coherent component. The ratio of the Fourier transforms of the transmitted and input signals gave the amplitude transmission coefficient.

To measure $I(\rho, t)$, a quasi-point source was created by focusing the pulsed ultrasonic beam onto a small aperture cut in the tip of an acoustically opaque cone-shaped screen. The cone shape was chosen so that edges of the beam could be effectively blocked when the aperture was placed close to the sample being investigated, while at the same time preventing significant stray sound being reflected back towards the sample from the screen. The transmitted field at a transverse distance $\rho$ was measured using a miniature hydrophone with a diameter of $400 \mu \mathrm{m}$, which is smaller than a wavelength, enabling the transmitted field to be detected in a single speckle spot. The average transmitted intensity $I(\rho, t)$ was determined for selected values of $\rho$ by scanning the sample position in a grid over a very large number of independent speckle spots $^{26}$. To measure the time-dependent transmission $I(t)$ for an extended quasi-plane-wave source, the sample was placed deep in the far field of a small-diameter planar transducer, and the transmitted speckle pattern was scanned by moving the hydrophone with the sample fixed in position. For measurements of both $I(\rho, t)$ and $I(t)$, the number of speckle spots over which the intensity was averaged was typically 529-3,025, and the bandwidth was set at $5 \%$ of the central frequency of the pulse by digitally filtering the transmitted field before determining the dynamic intensity profiles.

The normalized intensity $\hat{I} \equiv I /\langle I\rangle$ at a particular frequency was determined from the square of the magnitude of the Fourier transform of the transmitted field in each near-field speckle, normalized by the average intensity in the speckle pattern. The results were then binned to determine $P(\hat{I})$. To improve the statistics, the results were averaged over up to 100 frequencies within a $5 \%$ bandwidth where the statistics were similar-the same bandwidth as for the dynamic measurements of $I(\rho, t)$ and $I(t)$. In the localized regime, where fluctuations are largest, the statistical accuracy of the measured distribution was further improved by averaging over different samples, which were found to exhibit the same statistics. This enabled the error bars in Fig. $4 \mathrm{~d}$ to be determined from the standard error in the mean.

\section{Received 14 February 2008; accepted 10 September 2008; published 19 0ctober 2008}

\section{References}

1. Anderson, P. W. Absence of diffusion in certain random lattices. Phys. Rev. 109, 1492-1505 (1958).

2. Abrahams, E., Anderson, P. W., Licciardello, D. C. \& Ramakrishnan, T. V. Scaling theory of localization: Absence of quantum diffusion in two dimensions. Phys. Rev. Lett. 42, 673-676 (1979).

Vollhardt, D. \& Wölfle, P. Diagrammatic, self-consistent treatment of the Anderson localization problem in $d \leq 2$ dimensions. Phys. Rev. B 22, 4666-4679 (1980).

4. MacKinnon, A. \& Kramer, B. The scaling theory of electrons in disordered solids-additional numerical results. Z. Phys. B. 53, 1-13 (1983).

5. Kroha, J., Kopp, T. \& Wölfle, P. Self-consistent theory of Anderson localization for the tight-binding model with site-diagonal disorder. Phys. Rev. B 41, 888-891 (1990).

6. Dalichaouch, R., Armstrong, J. P. Schultz, S., Platzman, P. M. \& McCall, S. L. Microwave localization by 2-dimensional random scattering. Nature 354, 53-55 (1991).

7. Chabanov, A. A., Stoytchev, M. \& Genack, A. Z. Statistical signatures of photon localization. Nature 404, 850-853 (2000)

8. Wiersma, D. S., Bartolini, P., Lagendijk, A. \& Righini, R. Localization of light in a disordered medium Nature 390, 671-673 (1997)

9. Chabanov, A. A. Zhang, Z. Q. \& Genack, A. Z. Breakdown of diffusion in dynamics of extended waves in mesoscopic media. Phys. Rev. Lett. 90, 203903 (2003).

10. Störzer, M., Gross, P., Aegerter, C. M. \& Maret, G. Observation of the critical regime near Anderson localization of light. Phys. Rev. Lett. 96, 063904 (2006).

11. Aegerter, C. M., Störzer, M. \& Maret, G. Experimental determination of critical exponents in Anderson localisation of light. Europhys. Lett. 75, 562-568 (2006).

12. Schwartz, T., Bartal, G., Fishman, S. \& Segev, M. Transport and Anderson localization in disordered two-dimensional photonic lattices. Nature 446, 52-55 (2007).

13. Weaver, R. L. Anderson localization of ultrasound. Wave motion 12, 129-142 (1990).

14. Billy, J. et al. Direct observation of Anderson localization of matter waves in a controlled disorder. Nature 453, 891-894 (2008).

15. Roati, G. et al. Anderson localization of a non-interacting Bose-Einstein condensate. Nature 453, 895-899 (2008)

16. Chabé, J. et al. Experimental observation of the Anderson transition with atomic matter waves Preprint at $<$ http://arxiv.org/abs/0709.4320 > (2007).

17. Mott, N. Metal-insulator transitions. Phys. Today 31, 42-47 (1978).

18. John, S. Localization of light. Phys. Today 44, 32-40 (1991).

19. Anderson, P. W. The question of classical localization—a theory of white paint. Phil. Mag. B 52, 505-509 (1985)

20. Turner, J. A., Chambers, M. E. \& Weaver, R. L. Ultrasonic band gaps in aggregates of sintered aluminum beads. Acustica 84, 628-631 (1998).

21. Page, J. H. et al. Group velocity in strongly scattering media. Science 271, 634-637 (1996).

22. Cowan, M. L., Beaty, K., Page, J. H., Liu, Z. \& Sheng, P. Group velocity of acoustic waves in strongly scattering media: Dependence on the volume fraction of scatterers. Phys. Rev. E 58 6626-6635 (1998)

23. Schriemer, H. P., Pachet, N. G. \& Page, J. H. Ultrasonic investigation of the vibrational modes of a sintered glass-bead percolation system. Waves in Random Media 6, 361-386 (1996).

24. Van Tiggelen, B. A. Localization of waves. in Diffuse Waves in Complex Media (ed. Fouque, J. P.) 1-63 (Kluwer, 1998).

25. Scheffold, F., Lenke, R., Tweer, R. \& Maret, G. Localization or classical diffusion of light? Nature 398, 206-207 (1999).

26. Page, J. H., Schriemer, H. P., Bailey, A. E. \& Weitz, D. A. Experimental test of the diffusion-approximation for multiply scattered sound. Phys. Rev. E 52, 3106-3114 (1995)

27. De Raedt, H., Lagendijk, A. \& De Vries, P. Transverse localization of light. Phys. Rev. Lett. 62, $47-50$ (1989).

28. Van Tiggelen, B. A., Lagendijk, A. \& Wiersma, D. S. Reflection and transmission of waves near the localization threshold. Phys. Rev. Lett. 84, 4333-4336 (2000).

29. Skipetrov, S. E. \& Van Tiggelen, B. A. Dynamics of Anderson localization in open 3D media. Phys. Rev. Lett. 96, 043902 (2006)

30. Nieuwenhuizen, Th. M. \& Van Rossum, M. C. W. Intensity distributions of waves transmitted through a multiple scattering medium. Phys. Rev. Lett. 74, 2674-2677 (1995).

Acknowledgements

Financial support from NSERC of Canada and a CNRS France-Canada PICS project is gratefully acknowledged. The calculations in this work were carried out on the cluster HealthPhy (CIMENT, Grenoble)

Author information

Reprints and permissions information is available online at http://npg.nature.com/reprintsandpermissions. Correspondence and requests for materials should be addressed to J.H.P. 